\title{
Correction to: Identity Development in the Lifecourse
}

\section{Correction to:}

M. Märtsin, Identity Development in the Lifecourse, Sociocultural Psychology of the Lifecourse, https://doi.org/10.1007/978-3-030-27753-6

The original version of this book has been revised, An error in the production process caused the author's affiliation to be corrected into the series editor's affiliation by mistake. This has been corrected in the copyright page and the cover.

The updated version of the book can be found at https://doi.org/10.1007/978-3-030-27753-6 